\title{
Endoscopic Resection of a Large Polypoid Gastric Heterotopia of Duodenum: a case report
}

\author{
Francesco Maione ${ }^{1}$, Alessia Chini ${ }^{1}$, Nicola Gennarelli ${ }^{1}$, Marco Milone ${ }^{1}$, Michele \\ Manigrasso $^{1}$, Elia Guadagno ${ }^{1}$, Giovanni Aprea ${ }^{1}$, and Giovanni De Palma ${ }^{1}$ \\ ${ }^{1}$ University of Naples Federico II
}

June 4, 2021

\begin{abstract}
This is a case of gastric heterotopia, a benign condition most commonly found as multiple small polyps in the duodenal bulb, diagnosticated as a large polypoid lesion of the third duodenal portion in a young patient and treated successfully with endoscopic resection under laparoscopic vision.
\end{abstract}

\section{Endoscopic Resection of a Large Polypoid Gastric Heterotopia of Duodenum: a case report}

Francesco Maione ${ }^{1}$, Alessia Chini ${ }^{1}$, Nicola Gennarelli ${ }^{1}$, Marco Milone ${ }^{1}$, Michele Manigrasso ${ }^{2}$, Elia Guadagno $^{2}$, Giovanni Aprea ${ }^{1}$, Giovanni Domenico De Palma ${ }^{1}$

${ }^{1}$ Department of Clinical Medicine and Surgery, "Federico II" University of Naples, Via Sergio Pansini, 5, 80131, Naples, Italy

${ }^{2}$ Department of Advanced Biomedical Sciences, "Federico II" University of Naples, Via Sergio Pansini, 5, 80131, Naples, Italy

Correspondence to : Alessia Chini, MD. Department of Clinical Medicine and Surgery, "Federico II" University of Naples, Via Sergio Pansini, 5, 80131, Naples, Italy

E-mail:dr.alessiachini@gmail.com; +393292122828

\section{Abstract}

This is a case of gastric heterotopia, a benign condition most commonly found as multiple small polyps in the duodenal bulb, diagnosticated as a large polypoid lesion of the third duodenal portion in a young patient and treated successfully with endoscopic resection under laparoscopic vision.

Keywords: gastric heterotopia, endoscopy, duodenal lesion, endoscopic resection

Key clinical message

This report shows a rare case of gastric heterotopia in the duodenum presented as a large polypoid lesion, differently from data reported in literature, which enters into differential diagnosis with other duodenal lesions that are not always benign.

\section{Introduction}

Duodenal lesions are uncommon findings in patients submitting to esophagogastroduodenoscopy (EGD), with an incidence variable from less than $1 \%$ to $5 \%{ }^{1,2}$. 
The most common lesions requiring resection are duodenal adenomas, which should be differentiated from other mucosal lesions, such as heterotopic gastric mucosa, and submucosal lesions, such as carcinoids and gastrointestinal stromal tumours. Gastric heterotopia is a benign and often asymptomatic condition consisting on the presence of gastric tissue in a place where is not normally found and it has been described in several locations through the gastrointestinal tract. Gastric heterotopia in duodenum usually is an incidental finding and it is most commonly found as multiple small polyps in the duodenal bulb ${ }^{2}$. In this report, we describe a rare case of a patient with gastric heterotopia in the third duodenal portion, treated with endoscopic resection under laparoscopic vision.

\section{Case report}

A 20-year-old man, with a 4-year history of dyspeptic symptoms successfully treated with pharmacological therapy (low dose PPI), presented to a local hospital in January 2019 for recurrence of dyspepsia, vomiting and abdominal pain.

EGD revealed a submucosal polypoid lesion of about $2 \mathrm{~cm}$, with a short peduncle, in the third duodenal portion. Endoscopic ultrasound confirmed the presence of polypoid neoformation of the third duodenal portion, with subepithelial development and apparently confined to the mucosa, while magnetic resonance of the abdomen with contrast did not identify the lesion.

Physical examination and laboratory investigations were unremarkable.

The patient presented to our endoscopic unit and, after repetition of EGD, that showed the duodenal lesion unchanged compared to the previous procedure (Figure 1), he was hospitalized to remove the lesion endoscopically, under laparoscopic vision. The laparoscopic procedure began with pneumoperitoneum and placement of three ports, then an enterostat was placed at the first jejunal loop for the scopic control of the polypectomy. An endoscope with a front-view was used to reach the lesion located between the third and fourth duodenal portions. Saline solution and methylene blue was injected into the peduncle of the lesion, and the neoformation was resected en bloc using a snare cautery; hemoclips were placed at the base for additional hemostasis (Figure 1). The resected lesion measured approximately 2,6 x 1,5 cm and histopathological examination revealed heterotopic gastric mucosa in the duodenum (Figure 2). There were no complications related to the procedure. No residual tissue was observed on repeat endoscopic examination after 1 year and the patient remained asymptomatic.

\section{Discussion}

Duodenal lesions are a rare finding in patients submitting to upper endoscopy, with studies reporting rates from less than $1 \%$ to $5 \%^{1,2}$, even if their incidence has increased with the improvement of advanced endoscopic techniques and the widespread use of upper gastrointestinal endoscopy; most of duodenal lesions are detected incidentally during EGD, that is performed for other gastrointestinal issues.

Patients with duodenal lesions are often asymptomatic; when they occur with symptoms, these include dyspepsia, abdominal pain, obstruction, gastrointestinal bleeding. Duodenal lesions can be divided into subepithelial lesions, such as lipomas, gastrointestinal stromal tumours (GISTs) and carcinoids, and mucosallybased lesions, such as Brunner's gland adenomas or hamartomas, solitary Peutz-Jeghers polyps and ampullary or not-ampullary adenomas.

The diagnostic assessment of these lesions is important to guide the appropriate treatment: the visual endoscopic appearance allows to differentiate neoplastic lesions from others with no malignant potential by using magnification endoscopy, chromoendoscopy and high-definition narrow-band imaging (NBI) for the evaluation of morphological features and the pit pattern. Endoscopic ultrasonography is also an effective imaging modality that allows real-time assessment of a lesion's depth of invasion and nodal status, and the differentiation of sub-epithelial lesions.

Treatment depends on various factors, such as size, location and wheter it is subephitelial or mucosally-based; endoscopic resection of duodenal lesions provides a challenge due to certain anatomic characteristics of the 
duodenum, such as a narrow lumen, a thin wall and a rich vascularity, that result, respectively, in a higher rate of perforation and bleeding. Endoscopic management involves cold snare polypectomy for small lesions and endoscopic mucosal resection for larger mucosally-based lesions located in the duodenum: this technique consists, firstly, in a submucosal injection of sterile normal saline (or a mixture of saline and epinephrine 1:100000) around and underneath the lesion and successively in a hot-snare polipectomy ${ }^{3}$. EMR has been shown to be safe and effective in the treatment of duodenal lesions, as reported in several studies, but it can be associated to a substantial risk of complication, such as delayed bleeding, perforation, especially in case of large $(10-29 \mathrm{~mm})$ or giant $(>30 \mathrm{~mm})$ lesions, pancreatitis and stenosis ${ }^{4}$.

ESD is not recommended in the duodenum for the high rate of complications, both for the thin duodenal wall and the rich vascularity. In case of GISTs or other malignant lesions, they should be referred for surgical resection after endoscopic diagnosis and laparoscopy has been adopted with gradually increasing frequency in the management of these lesions ${ }^{3}$.

Gastric heterotopia consists on the presence of gastric tissue in a place where is not normally found; the most common locations are the esophagus and Meckel's diverticulum but it may be found in all the gastrointestinal tract $^{5}$.

Duodenal gastric heterotopia is a benign condition affecting from $0.5 \%$ to $2 \%$ of the population and it can be classified into congenital or acquired type.

The congenital type is likely due to an error in the differentiation of the endoderm during embryogenesis and it was initially defined as an elevated lesion of specialized oxyntic glands replacing the whole thickness of the duodenal mucosa. Successively, it was proposed that fundic-type mucosa could develop in the duodenum as a consequence of metaplastic change induced by peptic injuries and, in particular, by hypergastrinemia, as reported in patients with Zollinger Ellison syndrome or in patients using proton-pump-inhibitor (PPI) ${ }^{6,7}$. On the basis of literature, we cannot certainly say wheter the case of our patient is congenital or acquired; young age favours congenital origin, although the continued use of PPI cannot exclude acquired form. It's important to differentiate gastric heterotopia from gastric metaplasia, that is an acquired lesion consisting on a change of one type of fully developed tissue to another differentiated tissue, usually due to inflammatory conditions, where there are no oxyntic glands ${ }^{8}$.

Gastric heterotopia usually is an incidental finding and often it is asymptomatic, or it may presented with dyspepsia, peptic ulcer symptoms, gastrointestinal bleeding, perforation or, rarely with obstructive symptoms. In most cases, the diagnosis of gastric heterotopia is performed by histological examination and not during endoscopy, as in this report. Agha et al. ${ }^{5}$ described 17 cases of heterotopic gastric mucosa in the duodenal bulb confirmed by histological examination in 25 patients undergoing EGD; endoscopic features were related to the radiographic findings of the lesion on upper gastrointestinal barium studies and $68 \%$ of cases were described as clusters of 1 to $3 \mathrm{~mm}$ plaques raised above the healthy duodenal mucosa, and in $20 \%$ of cases as nodular mucosa with superficial erosions. The most frequent location is duodenal bulb, where gastric heterotopia is commonly found as multiple small polyps ${ }^{2}$ : to the best of our knowledge, this is the first report of a large polypoid gastric heterotopia in the third portion of duodenum, removed endoscopically, in a young man. Data regarding gastric heterotopia in the duodenum derive from small studies, mostly carried out several years ago, and from rare case reports, and the clinical significance, histopathological and functional characteristics remains mostly unknown.

The therapeutic options for gastric heterotopia includes endoscopic or surgical resection to prevent complications; the combination of clinical presentation, endoscopic evaluation and radiologic imaging is useful in making the diagnosis and guiding to the appropriate treatment ${ }^{8}$. The endoscopic management of duodenal lesions remains challenging, because of the high risk of complications, such as bleeding and perforation, due to the thin duodenal wall and the rich vascularity; furthermore, the curved anatomical configuration of the duodenum makes visualization and maintaining endoscope position difficult. For this reason, in this case we performed an EMR under laparoscopic vision in anticipation that it could be configured the impossibility of endoscopic management, both for the resection of the lesion and for the management of any complications 
requiring surgical treatment.

Conflicts of Interest : The authors have no potential conflicts of interest.

Funding: None.

Authors' contribution: FM, AC: conceptualized and designed the study, drafted the initial manuscript, and reviewed and revised the manuscript. NG, MM, MM: conducted literature search on the topic, contributed to writing of discussion and revised the manuscript. EG: led histopathology interpretation of case and revised the manuscript. GA, GDP: supervised work and revised the manuscript. All authors approved the final manuscript as submitted and agree to be accountable for all aspects of the work..

Acknowledgments : None to declare

\section{References}

1. Ghazi A, Ferstenberg H, Shinya H. Endoscopic gastroduodenal polypectomy. Ann Surg 1984; 200:175180

2. Jepsen JM, Persson M, Jakobsen NO et al. Prospective study of prevalence and endoscopic and histopathologic characteristics of duodenal polyps in patients submitted to upper endoscopy. Scand J Gastroenterol 1994; 29:483-487

3. Gaspar JP, Stelow EB, Wang AY. Approach to the endoscopic resection of duodenal lesions. World J Gastroenterol 2016; 22(2):600-617

4. Probst A, Freund S, Neuhaus L et al. Complication risk despite preventive endoscopic measures in patients undergoing endoscopic mucosal resection of large duodenal adenomas. Endoscopy 2020;52: $847-855$

5. Agha FP, Ghahremani GG, Tsang TK, Victor TA. Heterotopic gastric mucosa in the duodenum; radiographic findings. AJR Am J Roentgenol 1988;150:291-294

6. Conlon N, Logan E, Veerappan S, McKiernan S, O’Briain S. Duodenal gastric heterotopia: further evidence of an association with fundic gland polyps. Hum Pathol 2013;44:636-642

7. Genta RM, Kinsey RS, Singhal A, Suterwala S. Gastric foveolar metaplasia and gastric heteropia in the duodenum: no evidence of an etiologic role for Helicobacter pylori. Hum Pathol 2010;41:1593-600

8. Urquhart S.A., Coelho-Prabhu N. Jejunal Polyps out of Place: A Case of gastric Heterotopia of the Jejunum. Case Reports in Gastrointestinal Medicine, Vol. 2020

Figure legends: Figure 1) A: Endoscopy showing a neoformation with a short peduncle in the third duodenal portion. B: Saline solution with a drop of methylene blue is injected into the peduncle of the lesion. C: Neoformation is resected en bloc using a snare cautery. D: Endoscopy after one year from resection showing no residual tissue

Figure 2) Histopathological examination showing the mucosa of the small bowel partially replaced by gastric mucosa, with evident foci of foveolar epithelium and oxyntic glands, as expected in case of gastric heterotopia (Haematoxylin and eosin staining, a:100x; b: 200x and c: 400x) 

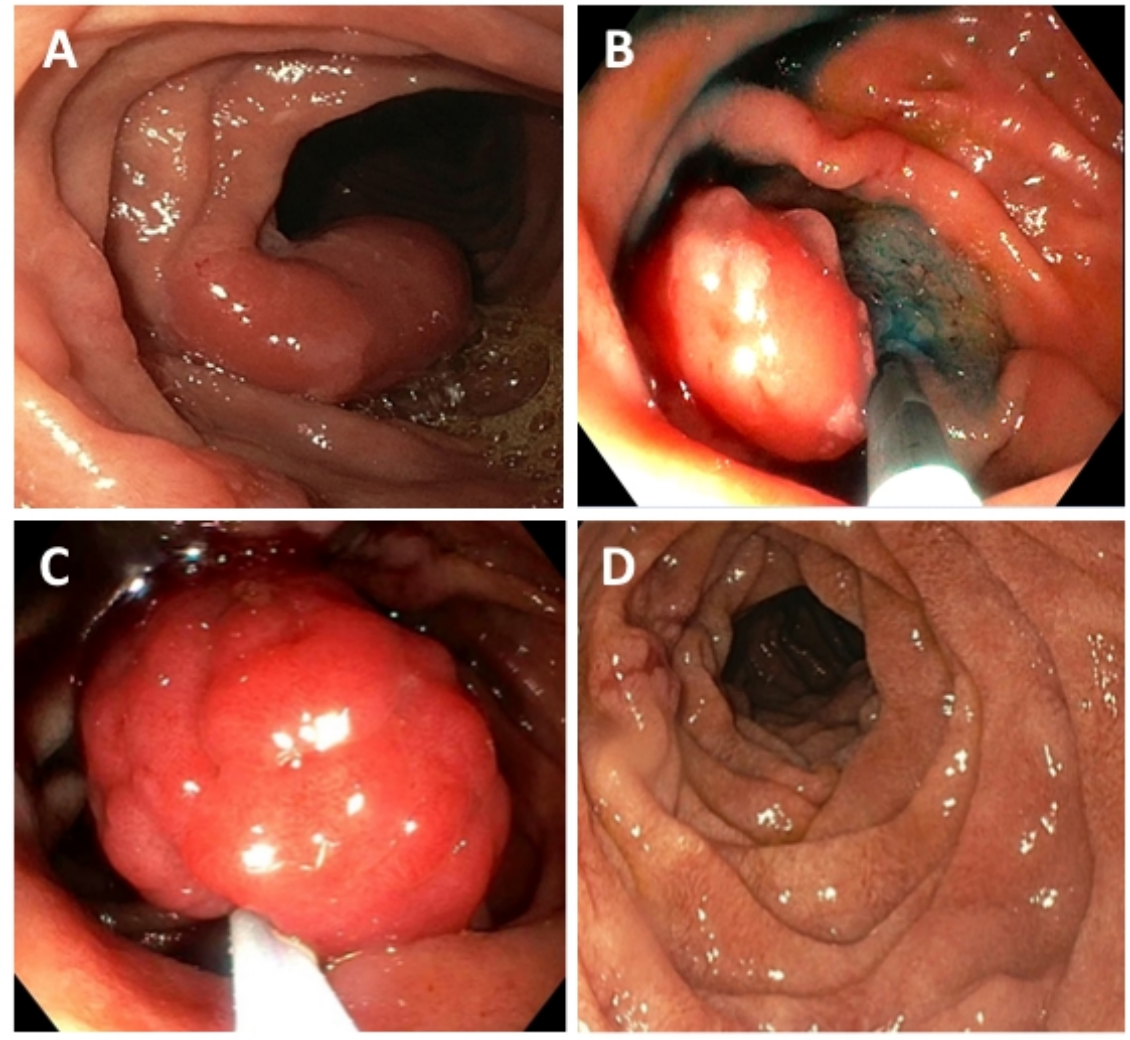

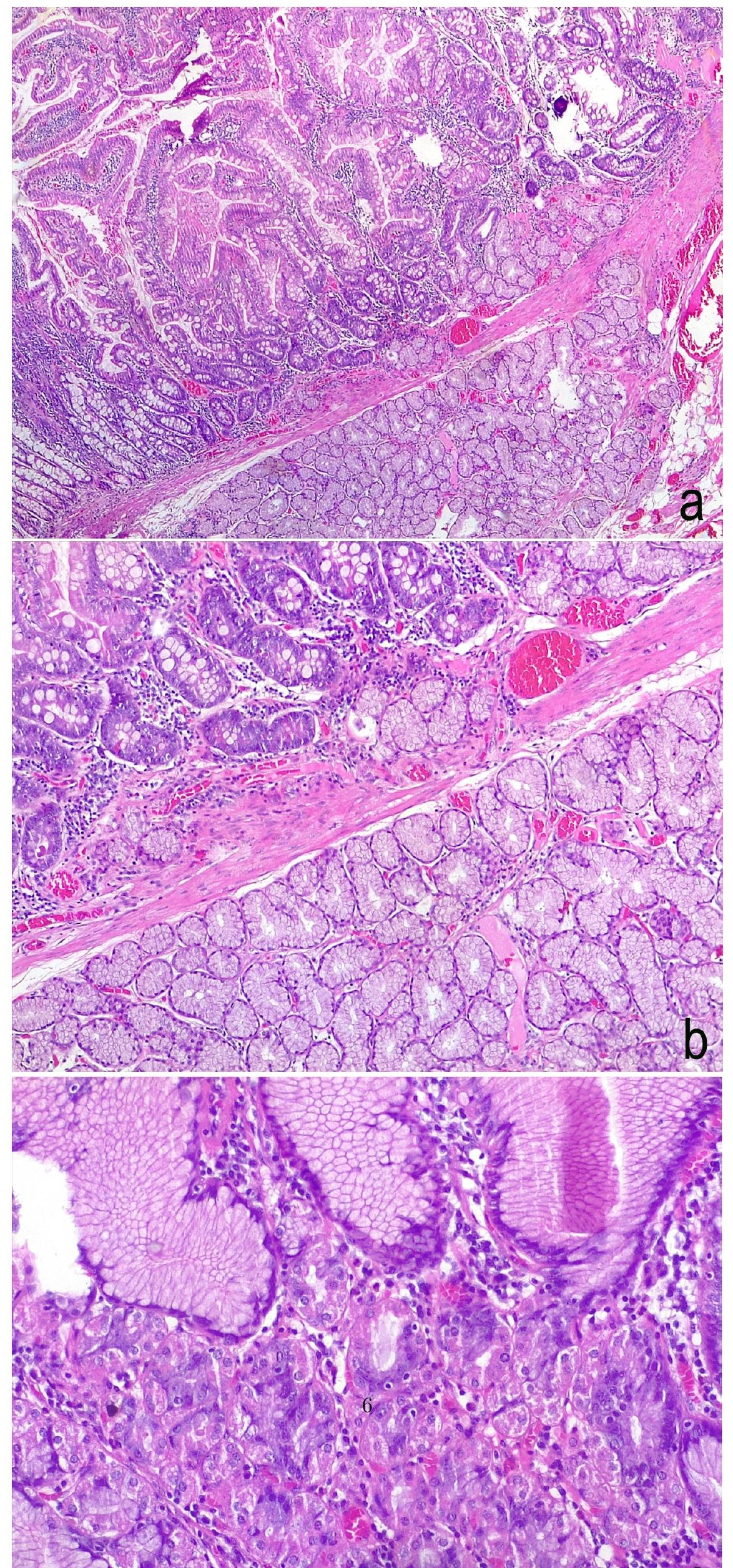